\title{
ORGASMIC DISORDER IN WOMEN
}

\author{
CRISTIAN DELCEA \\ Iuliu Hațieganu University of Medicine and Pharmacy, Cluj-Napoca, Romania \\ Sexology Institute of Romania, Cluj-Napoca, Romania
}

\begin{abstract}
Significant delay or absence of orgasm or reduced frequency during sexual intercourse with or without penetration / stimulation and / or after penetration. Markedly reduced intensity of orgasmic sensations and individual psychological discomfort. Women with orgasm disorder have never had a clitoral or vaginal orgasm (penetration) regardless situation or stimulation. Worldwide prevalence of orgasmic disorder in women is $42 \%$ and can be maintained depending on partner, stimulation, situation or regardless partner, stimulation, situation etc.The disorder may emerge from the beginning of the sexual life or begin after a period of relatively normal sexual function.
\end{abstract}

Keywords: orgasmic disorder in women, s-on, therapy, testing, evaluation, sexual disorders.

\section{INTRODUCTION}

One can book an appointment online, by phone, sms. You will receive confirmation of the appointment date and you will be asked to pay for the first intervention, after which you will take the necessary steps for the chosen activity. After payment and proof of payment you will go through each step below.

There will be a complex evaluation and testing with the S-ON Test $\mathbb{C}$ Clinical Sexual Assessment System.

After testing, you will complete the 7 standardized S-ON Sextherapy@ protocols to address sexual and / or couple issues.

The next step is using S-ON Monitoring® for monitoring and feedback throughout the entire period of interventions to improve orgasmic disorder in women as well as S-ON Optimization $($ to optimize sexual performance and couple's relationship.

You will receive more details during our interventions.
How do I pay for the service and how much does it cost?

The payment is made online into the account of Institute of Sexology: bank account: RO45BTRL06701205M34615XX opened at Banca Transilvania. And the cost for each intervention (evaluation, testing, intervention protocol) is 100 euro at the NBR (National Bank of Romania) exchange rate.

Let's start!

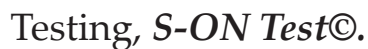

Protocols, S-ON Sextherapy@

Monitoring, S-ON Monitoring $₫$

Optimizing, S-ON Optimization@

\section{APPLICATIONS}

\section{- Testing, S-ON Test $\odot$ WOMEN} Screening-DSM/Of (S-DSM/Of)

INSTRUCTIONS. You will find below a checklist of 8 questions that describe the actions that women take in various sexual

*Corresponding author: 160 Plevnei Street, Cluj-Napoca, 400000, Romania, Phone/Fax: 0264 550247, email cristian.delcea.cj@gmail.com 
intercourses. For each question, check the option that best suits you.

1/8 Do you have difficulties in reaching an orgasm or you are unable to have an orgasm after adequate sexual stimulation and penetration?

Not at all

A little

A lot

Very much

Extremely

2/8 6 or 7 out of 10 sexual intercourses have a reduced intensity of orgasmic sensations?

Not at all

A little

A lot

Very much

Extremely

3/8 Has the orgasmic disorder occurred since the beginning of your sexual life?

Not at all

A little

A lot

Very much

Extremely

4/8 Has orgasmic disorder appeared after a long time, reported to the beginning of sexual life?

Not at all

A little

A lot

Very much

Extremely

5/8 Does orgasmic disorder occur when you are with your stable long-term partner?

Not at all

A little

A lot

Very much

Extremely

6/8 Does it appear irrespective of the partner? Not at all

A little
A lot

Very much

Extremely

$7 / 8$ Is there an anticipatory fear of a new sexual failure?

Not at all

A little

A lot

Very much

Extremely

8/8 Do you have couple issues regarding sexual intercourses?

Not at all

A little

A lot

Very much

Extremely

Answers

- Not at all, 0 percentages.

- A little, 10 percentages, MILD orgasm disorder is confirmed. This means you have signs and symptoms of marked difficulty in obtaining orgasm.

- A lot, 20 percentages, MODERATE orgasm disorder is confirmed. This means you have signs and symptoms of marked difficulty in obtaining orgasm or the absence of it.

- Very much, 30 percentages, SEVERE orgasm disorder is confirmed. This means you have signs and symptoms of marked difficulty in obtaining orgasm or total absence of it.

- Extremely, 40 percentages, EXTREMELY SEVERE orgasm disorder is confirmed. This means you have signs and symptoms of marked difficulty in obtaining orgasm or total absence of it.

\section{- Testing, S-ON Test@ WOMEN General clinical sexual screening GSCS-w}

INSTRUCTIONS. You will find below a checklist of 36 questions that describe the actions that men take in various sexual intercourses. For each question, check the option that best suits you. 
$1 / 33$ Is there a significant delay in orgasm in approximately $75-100 \%$ of sexual intercourses occasions?

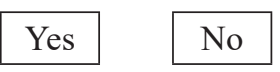

2/33 Is there a reduced intensity of orgasmic sensations in approximately $75-100 \%$ of sexual intercourses?

Yes No

$3 / 33$ Is there a significant reduction of sexual arousal in approximately $75-100 \%$ of sexual intercourses?

Yes No

4/33 Is there a significant reduction of sexual arousal in response to any erotic stimuli in approximately $75-100 \%$ of sexual intercourses?

Yes No

$5 / 33$ Is there a significant reduction of genital sensations or erogenous zones in approximately $75-100 \%$ of sexual intercourses? Yes No

6/33 Is there a significant lack of sexual desire?

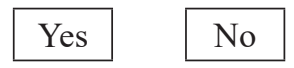

$7 / 33$ Is there a significant lack of arousal and sexual orgasm?

Yes No

$8 / 33$ Is there a significant reduced interest in sexual activity?

Yes No

9/33 Is there a significant reduction in erotic thoughts/ fantasies?

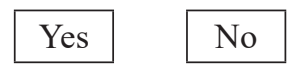

10/33 Is there a persistent / recurrent discomfort with penetration?

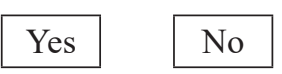

11/33 Is there a persistent / recurrent pain with penetration?

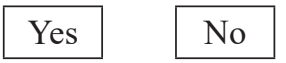

$12 / 33$ Is there an intense fear/anxiety related to pain/discomfort occurence with penetration?

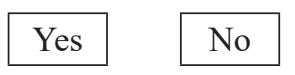

$13 / 33$ Is there a significant strain / contraction of the pelvic floor muscles with penetration?

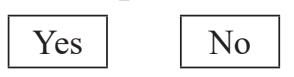

14/33 Your partner doesn't sexually satisfy you?

Yes No

$15 / 33$ Is there a personality incompatibility with your partner?

Yes No

16/33 You and your partner argue over sexual matters?

Yes No

17/33 You and your partner don't share the same level of sexual interest?

Yes No

18/33 Do you feel uncomfortable engaging in some sexual activities that your partner wants

Yes No

$19 / 33$ When it comes to sex, you have different ideas and values from your partner?

Yes No

20/33 Do you think you don't meet the sexual needs of your partner?

Yes No

21/33 Do you and think that you and your partner don't enjoy the same sexual activities

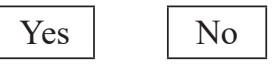

22/33 When it comes to sex, you and your partner don't get along well?

Yes No

23/33 Do you find it's difficult for you to accept your partner's perspective on sex?

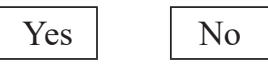


24/33 You and your partner don't agree on how often you should have sex?

Yes No

25/33 You and your partner don't share similar sexual fantasies?

Yes No

26/33 When it comes to sex, your partner your partner isn't willing to do certain things that you would like to do?

Yes No

27/33 You and your partner don't share the same level of sexual desire?

Yes No

28/33 Do you think that your partner doesn't understand you from sexual point of view?

Yes No

29/33 You and your partner share the same sexual preferences?

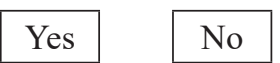

30/33 Isn't your partner willing to do certain sexual things that you would like to do

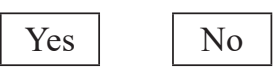

31/33 Do you feel comfortable during sex with your partner?

Yes No

32/33 Do you consider yourself sexually inhibited by your partner?

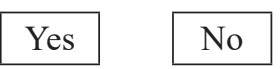

33/33 You and your partner aren't sexually attracted to each other?

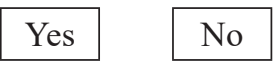

\section{Answers}

- Orgasmic disorder is confirmed $(1,2,7)$

- Arousal disorder is confirmed $(3,4,5)$

- Desire disorder is confirmed $(6,8,9)$

- Dyspareunia is confirmed $(10,11,12,13)$

- Couple issues are confirmed (14-33)
- Testing, S-ON Test $@$ WOMEN

Couple satisfaction scale CSSw

INSTRUCTIONS. You will find below a checklist of 30 questions that describe the actions that women take in various sexual intercourses. For each question, check the option that best suits you.

$1 / 30$ Spending free time together is beneficial for your relationship

A little

A lot

Very much

2/30 Emotional support is sufficiently adaptive A little

A lot

Very much

3/30 Your partner hardly ever responds when you want to discuss your sex life

A little

A lot

Very much

$4 / 30$ Your sexual life is satisfied

A little

A lot

Very much

$5 / 30$ Some sexual issues are too uncomfortable to discuss with your partner

A little

A lot

Very much

6/30 You and your partner don't seem to resolve your misunderstandings on sexual matters

A little

A lot

Very much

$7 / 30$ There is enough time invested in the relationship

A little

A lot

Very much 
$8 / 30$ There are sexual issues in your relationship that you have never discussed

A little

A lot

Very much

9/30 Making important decisions together is satisfactory

A little

A lot

Very much

10/30 When you and your partner talk about sex, you feel he is criticizing you

A little

A lot

Very much

11/30 You have shared hobbies, interests, activities

A little

A lot

Very much

$12 / 30$ You have common activities or interests outside the couple/family

A little

A lot

Very much

13/30 Your partner often complains that you're not clear enough about what you want from a sexual point of view

A little

A lot

Very much

14/30 You laugh and smile together

A little

A lot

Very much

15/30 You work together to project, business, etc.

A little

A lot

Very much

$16 / 30$ You show your love and you say you love each other

A little

A lot

Very much

17/30 Your partner has no difficulty talking to you about his feelings or desires

A little

A lot

Very much

$18 / 30$ Even when he is furious with you, your partner is able to appreciate your perspectives on sexuality

A little

A lot

Very much

19/30 You and your partner have never had an open discussion about your sex life

A little

A lot

Very much

20/30 You have common friends

A little

A lot

Very much

21/30 You know each other well enough

A little

A lot

Very much

22/30 You tolerate each other's little/ big naughty things

A little

A lot

Very much

23/30 You have secrets

A little

A lot

Very much

24/30 You are independent in your couple relationship

A little

A lot 
Very much

25/30 Talking about sex is a satisfying experience for both of you

A little

A lot

Very much

26/30 You and your partner can usually talk calmly about your sex life

A little

A lot

Very much

27/30 You have a slight difficulty in telling your partner what you're doing and what you're not doing from sexual point of view

A little

A lot

Very much

28/30 You rarely feel ashamed when discussing details about your sex life

A little

A lot

Very much

29/30 You are dependent on one another

A little

A lot

Very much

30/30 How attached you are to each other

A little

A lot

Very much

\section{Answers}

- A little, 10 percentages: You obtained a low level with regard to your couple tolerance to vital factors for relationship, such as time spent together, your money, hobbies, friendships, sex life, common interests, shared activities, individual satisfaction towards expressing affection and relationship intimate life as well as your commitment to continuing the relationship.

- A lot, 20 percentages: You obtained a low level with regard to your couple tolerance to vital factors for relationship, such as time spent together, your money, hobbies, friendships, sex life, common interests, shared activities, individual satisfaction towards expressing affection and relationship intimate life as well as your commitment to continuing the relationship;

- Very much, 30 percentages: You obtained a low level with regard to your couple tolerance to vital factors for relationship, such as time spent together, your money, hobbies, friendships, sex life, common interests, shared activities, individual satisfaction towards expressing affection and relationship intimate life as well as your commitment to continuing the relationship.

\section{- Testing, S-ON Test $\odot$ WOMEN Cognito-sexual questionnaire CSQ-w}

INSTRUCTIONS. You will find below a checklist of 15 questions that describe the actions of women in various sexual intercourse. For each question, check the option that best suits you.

1/15 What do you THINK of during intercourse regarding what you hear?

1. Sounds of your partner's hyperventilation (inhalation and exhalation acceleration), the sound of movements and other auditory sensations

2. Your partner verbalizing words with sexual connotations

3. A part of the first two options above

4. You don't give too much importance to auditory sensations

\section{2/15 What doyou THINK of during intercourse} regarding what you see

1. Intimate positions that will allow you to see your partner during intercourse and your partner's body

2. Your partner's body

3. A part of the first two options above

4. You don't give too much importance to visual sensations 
3/15 What do you THINK of during intercourse regarding what you smell

1. Your partner's body

2. On some parts of your partner's body

3. A part of the first two options above

4. You don't give too much importance to smell and taste sensations

4/15 What do you THINK of during intercourse regarding what you touch

1. You focus on details when touching your partner (focus on face, neck, breasts, abdomen, penis, buttocks, legs, back, arms)

2. You focus on overall caresses (you focus only on some parts of your partner's body)

3. A part of the first two options above

4. You don't give too much importance to tactile sensations

5/15 What do you THINK of during intercourse regarding your moves

1. You practice more positions (classic missionary, cowgirl, doggy-style, etc.) in a sexual intercourse

2. You practices 1 or maximum two positions (classic missionary, cowgirl, doggy-style, etc.) in a sexual intercourse

3. Your sexual activity is more dinamic and with few moves

4. You don't give too much importance to your moves during intercourse

6/15 What do you FEEL during intercorse regarding what you hear

1. Hearing sensations that produce well-being, pleasure and excitement

2. Hearing sensations that produce excitement and pleasure

3. Hearing sensations that produce pleasure

4. Hearing sensations that don't produce well-being, pleasure and excitement

7/15 What do you FEEL during intercorse regarding what you see

1. Visual sensations that produce well-being, pleasure and excitement

2. Visual sensations that produce excitement and pleasure

3. Visual sensations that produce pleasure
4. Visual sensations that don't produce well-being, pleasure and excitement

8/15 What do you FEEL during intercorse regarding what you touch

1. Tactile sensations that produce well-being, pleasure and excitement

2. Tactile sensations that produce excitement and pleasure

3. Tactile sensations that produce pleasure

4. Tactile sensations that don't produce well-being, pleasure and excitement

9/15 What do you FEEL during intercorse regarding what you smell

1. Taste and smell sensations that produce well-being, pleasure and excitement

2. Taste and smell sensations that produce excitement and pleasure

3. Taste and smell sensations that produce pleasure

4. Taste and smell sensations that don't produce well-being, pleasure and excitement

10/15 What do you FEEL during intercorse regarding your moves

1. Motion sensations that produce well-being, pleasure and excitement

2. Motion sensations that produce excitement and pleasure

3. Motion sensations that produce pleasure

4. Motion sensations that don't produce well-being, pleasure and excitement

11/15 What do you DO during intercorse regarding what you see

1. You insist on watching your partner and you use your imagination when you can't see parts of the body

2. You prefer positions that will allow you to see your partner during intercourse, to the detriment of those that don't allow you to do this

3. Your eyes are guided only by sensations

4. You don't use too much visual senses during intercourse

12/15 What do you DO during intercorse regarding what you hear

1. You prefer to communicate during sexual intercourse for sexual dinamics 
2. You are involved in communication during intercourse

3. You let yourself being driven by erotic words during intercourse

4. You are involved in what you hear

13/15 What do you DO during intercorse regarding what you touch

1. Your touches are made in successive steps (you touch all the body parts even the insignificant ones)

2. Your touches are short and frequent (meaning "more quickly", "in a hurry", "on the run")

3. Your touches are only made on parts of the body with maximum excitement

4. You don't touch too much during intercourse

14/15 What do you DO during intercorse regarding what you smell

1. You insist to smell parts of your partner's body (all parts of the body with erotic significance)

2. Sometimes you can smell parts of your partner's body (some parts of the body with erotic significance)

3. You stimulate olfactory and gustative senses during intercourse

4. You usually do nothing regarding smell

15/15 What do you DO during intercorse regarding your moves

1. Your movements are acurate and consistent (good management of movements during intercourse)

2. Your movements are balanced (a good style of having sex)

3. You have no control over movements (you can't find the "place" and "the right time" to have sex)

4. You are passive during intercourse (you prefer man in dominant role)

\section{Answers}

Mild: Your cognitive-behavioral involvement in excitatory stimuli is maladaptive. Your thinking, sensation and behavioral process is not well-managed/ adaptively used.
- Moderate: Your cognitive-behavioral involvement in excitatory stimuli is maladaptive. Your thinking, sensation and behavioral process is not sufficiently managed/ adaptively used.

- Severe: Your cognitive-behavioral involvement in excitatory stimuli is maladaptive. Your thinking, sensation and behavioral process is insufficient concerning the adaptive management.

\section{- Testing, S-ON Test@ MEN Sexual stimuli genogram SSG-m}

INSTRUCTIONS. You will find below a set of 3 questions that describe the actions that men do in various sexual acts. For each question, check the option that best suits you. Choose the answer you think is most appropriate.

You must tick from 1-10 the intensity of pleasure (sexual attraction), relaxation (sexual disposition) and arousal (sexual stimulation).

These ticked grades, in terms of pleasure, relaxation and arousal are the category of stimuli that you work with during the sexual intercourse. You have to think carefully about what you choose to identify your real answer.

1. PLEASURE (the extent to which you feel attracted by stimuli below)

Face

\begin{tabular}{|l|l|l|l|l|l|l|l|l|l|}
\hline 1 & 2 & 3 & 4 & 5 & 6 & 7 & 8 & 9 & 10 \\
\hline
\end{tabular}

Neck

\begin{tabular}{|l|l|l|l|l|l|l|l|l|l|}
\hline 1 & 2 & 3 & 4 & 5 & 6 & 7 & 8 & 9 & 10 \\
\hline
\end{tabular}

Chest

\begin{tabular}{|l|l|l|l|l|l|l|l|l|l|}
\hline 1 & 2 & 3 & 4 & 5 & 6 & 7 & 8 & 9 & 10 \\
\hline
\end{tabular}

Abdomen

\begin{tabular}{|l|l|l|l|l|l|l|l|l|l|}
\hline 1 & 2 & 3 & 4 & 5 & 6 & 7 & 8 & 9 & 10 \\
\hline
\end{tabular}

Arms

\begin{tabular}{|l|l|l|l|l|l|l|l|l|l|}
\hline 1 & 2 & 3 & 4 & 5 & 6 & 7 & 8 & 9 & 10 \\
\hline
\end{tabular}

Penis

\begin{tabular}{|l|l|l|l|l|l|l|l|l|l|}
\hline 1 & 2 & 3 & 4 & 5 & 6 & 7 & 8 & 9 & 10 \\
\hline
\end{tabular}

Buttocks

\begin{tabular}{|l|l|l|l|l|l|l|l|l|l|}
\hline 1 & 2 & 3 & 4 & 5 & 6 & 7 & 8 & 9 & 10 \\
\hline
\end{tabular}




\section{Legs}

\begin{tabular}{|l|l|l|l|l|l|l|l|l|l|}
\hline 1 & 2 & 3 & 4 & 5 & 6 & 7 & 8 & 9 & 10 \\
\hline
\end{tabular}

Back

\begin{tabular}{|l|l|l|l|l|l|l|l|l|l|}
\hline 1 & 2 & 3 & 4 & 5 & 6 & 7 & 8 & 9 & 10 \\
\hline
\end{tabular}

2. RELAXATION (the extent to which you enjoy the stimuli below)

Face

\begin{tabular}{|l|l|l|l|l|l|l|l|l|l|}
\hline 1 & 2 & 3 & 4 & 5 & 6 & 7 & 8 & 9 & 10 \\
\hline
\end{tabular}

Neck

\begin{tabular}{|l|l|l|l|l|l|l|l|l|l|}
\hline 1 & 2 & 3 & 4 & 5 & 6 & 7 & 8 & 9 & 10 \\
\hline
\end{tabular}

Chest

\begin{tabular}{|l|l|l|l|l|l|l|l|l|l|}
\hline 1 & 2 & 3 & 4 & 5 & 6 & 7 & 8 & 9 & 10 \\
\hline
\end{tabular}

Abdomen
\begin{tabular}{|l|l|l|l|l|l|l|l|l|l|}
\hline 1 & 2 & 3 & 4 & 5 & 6 & 7 & 8 & 9 & 10 \\
\hline
\end{tabular}

Arms

\begin{tabular}{|l|l|l|l|l|l|l|l|l|l|}
\hline 1 & 2 & 3 & 4 & 5 & 6 & 7 & 8 & 9 & 10 \\
\hline
\end{tabular}

\section{Penis}

\begin{tabular}{|l|l|l|l|l|l|l|l|l|l|}
\hline 1 & 2 & 3 & 4 & 5 & 6 & 7 & 8 & 9 & 10 \\
\hline
\end{tabular}

\section{Buttocks}

\begin{tabular}{|l|l|l|l|l|l|l|l|l|l|}
\hline 1 & 2 & 3 & 4 & 5 & 6 & 7 & 8 & 9 & 10 \\
\hline
\end{tabular}

\section{Legs}

\begin{tabular}{|l|l|l|l|l|l|l|l|l|l|}
\hline 1 & 2 & 3 & 4 & 5 & 6 & 7 & 8 & 9 & 10 \\
\hline
\end{tabular}

Back

\begin{tabular}{|l|l|l|l|l|l|l|l|l|l|}
\hline 1 & 2 & 3 & 4 & 5 & 6 & 7 & 8 & 9 & 10 \\
\hline
\end{tabular}

3 AROUSAL (the extent to which you are stimulated/aroused by stimuli below)

Face

\begin{tabular}{|l|l|l|l|l|l|l|l|l|l|}
\hline 1 & 2 & 3 & 4 & 5 & 6 & 7 & 8 & 9 & 10 \\
\hline
\end{tabular}

Neck

\begin{tabular}{|l|l|l|l|l|l|l|l|l|l|}
\hline 1 & 2 & 3 & 4 & 5 & 6 & 7 & 8 & 9 & 10 \\
\hline
\end{tabular}

\section{Chest}

\begin{tabular}{|l|l|l|l|l|l|l|l|l|l|}
\hline 1 & 2 & 3 & 4 & 5 & 6 & 7 & 8 & 9 & 10 \\
\hline
\end{tabular}

\section{Abdomen}

\begin{tabular}{|l|l|l|l|l|l|l|l|l|l|}
\hline 1 & 2 & 3 & 4 & 5 & 6 & 7 & 8 & 9 & 10 \\
\hline
\end{tabular}

\section{Arms}

\begin{tabular}{|l|l|l|l|l|l|l|l|l|l|}
\hline 1 & 2 & 3 & 4 & 5 & 6 & 7 & 8 & 9 & 10 \\
\hline
\end{tabular}

\section{Penis}

\begin{tabular}{|l|l|l|l|l|l|l|l|l|l|}
\hline 1 & 2 & 3 & 4 & 5 & 6 & 7 & 8 & 9 & 10 \\
\hline
\end{tabular}

\section{Buttocks}

\begin{tabular}{|l|l|l|l|l|l|l|l|l|l|}
\hline 1 & 2 & 3 & 4 & 5 & 6 & 7 & 8 & 9 & 10 \\
\hline
\end{tabular}

Legs
\begin{tabular}{|c|c|c|c|c|c|c|c|c|c|}
\hline 1 & 2 & 3 & 4 & 5 & 6 & 7 & 8 & 9 & 10 \\
\hline Back \\
\hline 1 & 2 & 3 & 4 & 5 & 6 & 7 & 8 & 9 & 10 \\
\hline
\end{tabular}

\section{Answers}

- 5-7 percentages, Mild: Your cognitive involvement and utilization of excitatory, pleasure and relaxation stimuli is maladaptive. The number of excitatory stimuli is reduced (you are focused only on two or four excitatory stimuli maximum) and your sexual behavior is guided only towards those unrepresentative stimuli, failing control sexual intercourse.

- 3-5 percentages, Moderate: Your cognitive involvement and utilization of excitatory, pleasure and relaxation stimuli is maladaptive. The number of excitatory stimuli is very reduced (you are focused only on one or maximum three excitatory stimuli) and your sexual behavior is guided only towards those unrepresentative stimuli, failing control sexual intercourse

- 1-3 percentages, Severe: Your cognitive involvement and utilization of excitatory, pleasure and relaxation stimuli is maladaptive. The number of excitatory stimuli is extremely reduced (you are focused only on one or maximum two excitatory stimuli) and your sexual behavior is guided only towards those unrepresentative stimuli, failing control sexual intercourse.

\section{- Interventions, S-ON Sextherapy® Women Protocol of sexual cognitive scenario, $\mathrm{S}-\mathrm{ONo}$ cognitive $\odot$ Orgasm}

\section{General consideration}

The protocol of $S-O N d$ cognitive $@$ sexual cognitive scenario was scientifically validated with the purpose of helping women to develop a cognitive-behavioural participation by insisting on sexual stimulus, thus controlling and maintaining the orgasm. This technique uses cognitive scenarios and several steps to 
help women rediscover how to be involved at a cognitive-behavioural level by using relevant sexual stimulus for orgasm. In fact, it is a self guidance in how to participate at the cognitivebehavioural level only to the excitation stimulus by using descriptions and following certain rules: What am I doing? How am I doing it? and What am I going to do? Or What am I doing simultaneously?, so that you can pay attention to the maximum excitation/relevant stimulus.

\section{Focus}

This technique helps men to relearn how to think using all relevant sexual stimulus and how to manage the orgasm sensations. In fact, this protocol helps men to think ",analytically" in order to identify the excitation stimulus which help achieving and maintaining the orgasm.

\section{Specialist advice}

Are you familiar with a flight deck? If the answer is no, than ask the co-pilot! Substituting your attention on what you do rather than what you feel together with multitasking on several sexual stimulus, will help you to become an expert in sexual activity and to increase and maintain your sexual desire. This is what happens with an airplane pilot. Are you the pilot of the bed?

\section{Applications}

Make a description using internal monologue in order to cover step by step your partner's body by following these rules: What am I doing? How am I doing it? and What am I going to do? Or What am I doing simultaneously? and thus you can pay attention to what you do instead of how you feel.

Rule. You have the following exercises. First step, arousal (kisses), make a silent description of WHAT YOU DO (for instance, describe using internal monologue: "I start kissing the lips"). Then, carry on with the description, HOW YOU DO THAT (for instance, describe using internal monologue: "I kiss the lower lip, the upper lip, I touch her tongue and feel her tongue in my mouth"). Then, continue describing, WHAT YOU DO NEXT or WHAT YOU DO SIMULTANEOUSLY (for instance, describe using internal monologue: "I start kissing down the neck and both sides of the neck while I am playing with his penis"). Carry on in that way with the description for all stimulus/ areas of your partner!

Follow the example from the Table 1.

Table 1. Description for all stimulus/areas of your partner

\begin{tabular}{|l|l|}
\hline FACE & $\begin{array}{l}\text { I start kissing the face, gently touching it with my lips, then I touch it, and then..., } \\
\text { etc., ... }\end{array}$ \\
\hline NECK & $\begin{array}{l}\text { I start kissing the neck, gently touching it with my lips, then I touch it, and then..., } \\
\text { etc., . }\end{array}$ \\
\hline BREASTS & $\begin{array}{l}\text { I start kissing the breasts, gently touching them with my lips, then I touch and } \\
\text { slowly pull them, rubb and pinch them, and then..., etc., ... }\end{array}$ \\
\hline ABDOMEN & $\begin{array}{l}\text { I start kissing the abdomen, gently touching it with my lips, then I touch it, rubb it, } \\
\text { slowly pinch it, and then..., etc., ... }\end{array}$ \\
\hline BACK & $\begin{array}{l}\text { I start kissing the back, gently touching it with my lips, then I touch it, rubb it, slowly } \\
\text { pinch it, then..., etc., ... }\end{array}$ \\
\hline ARMS & $\begin{array}{l}\text { I start kissing the arms, gently touching them with my lips, then I touch them, slowly } \\
\text { pull them, rubb and pinch them, and then..., etc., ... }\end{array}$ \\
\hline VAGINA & $\begin{array}{l}\text { I start kissing the vagina, gently touching it with my lips, slowly pull it, I insert my } \\
\text { finger into vagina, I rubb it and pinch the labia, etc., ... }\end{array}$ \\
\hline BUTTOCKS & $\begin{array}{l}\text { I start kissing the buttocks, gently touching them with my lips, then I touch them } \\
\text { and slowly pull them, rubb and pinch them, and then..., etc., ... }\end{array}$ \\
\hline LEGS & $\begin{array}{l}\text { I start kissing the legs, then I touch them and slowly, pull them, rubb and pinch } \\
\text { them, and then..., etc., ... }\end{array}$ \\
\hline
\end{tabular}




\section{Well done!}

You have succeeded to complete the Protocol of sexual cognitive scenario S-ONo cognitive@. I know that this was something new for you and I hope that you have learnt how to ",analytically" think in order to manage the excitation stimulus and to achieve and maintain the orgasm.

\section{Feedback}

Was the Protocol of sexual cognitive scenario S-ONo cognitive $@$ useful for you? Please express your content by crossing one of the statements bellow:

1. Unsatisfactory

2. Satisfactory

3. Good

4. Very good

5. Excellent

Funding Sources: This research did not receive any specific grant from funding agencies in the public, commercial, or not-for-profit sectors.

\section{REFERENCES}

1. Abel, G. G., Becker, J. B., CunninghamRathner, J., Mittelman, M., \& Rouleau, J. L., 1988. Multiple paraphilic diagnoses among sex offenders. Bulletin of the American Academy of Psychiatry and the Law, 16, 153-168.

2. Abel, G. G., Becker, J. B., Mittelman, M., Cunningham-Rathner, J., Rouleau, J. L., \& Murphy, W. D., 1987. Self-reported sex crimes of nonincarcerated paraphiliacs. Journal of Interpersonal Violence, 2, 3-25.

3. Bain, J., Langevin, R., Dickey, R., \& Ben-Aron, M., 1987. Sex hormones in murderers and assaulters. Behavioral Sciences and the Law, 5, 95-101.

4. Breslow, N., Evans, N., \& Langley, J., 1985. On the prevalence and roles of females in sadomasochistic sub-culture: Report of an empirical study. Archives of Sexual Medicine, 14, 303-317.

5. Dietz, P., Hazelwood, R. R., \& Warren, J., 1990. The sexually sadistic criminal and his offenses. Bulletin of the American Academy of Psychiatry and the Law, 18, 163-178.

6. Fedora, O., Reddon, J. R., Morrison, J. W., Fedora, S. K., Pascoe, H., \& Yeudall, C. T., 1992. Sadism and other paraphilias in normal controls and aggressive and nonaggressive sex offenders. Archives of Sexual Behavior, 21, $1-15$.

7. Freud, S., 1961. On sexuality. Markham, ON: Penguin.

8. Fromm, E., 1977. The anatomy of human destructiveness. Markham, ON: Penguin.

9. Graber, B., Hartmann, K., Coffman, J., Huey, C., \& Golden, C., 1982. Brain damage among mentally disordered sex offenders. Journal of Forensic Sciences, 27, 127-134.

10. Gratzer, T., \& Bradford, J., 1995. Offender and offense characteristics of sexual sadists: A comparative study. Journal of Forensic Sciences, 40, 450-455.

11. Holmes, R. M., \& Holmes, S. T., 1994. Murder in America. Thousand Oaks, CA: Sage.

12. Hucker, S. J., 1990. Necrophilia and other unusual paraphilias. In R. Bluglass \& P. Bowden (Eds.), Principles and practice of forensic psychiatry (pp. 723-728). London: Churchill Livingstone.

13. Hucker, S. J., Langevin, R., Wortzman, G., Dickey, R., Bain, J., Jandy, L., et al., 1988. Cerebral damage and dysfunction in sexually aggressive men. Annals of Sex Research, 1, 3347.

14. Knight, R., Prentky, R. A., \& Cerce, D. D., 1994. The development, reliability, and validity of an inventory for the multidimensional assessment of sex and aggression. Criminal Justice and Behavior, 21, 72-94.

15. Laws, D. R., \& O’Donohue, W., 1997. Fundamental issues in sexual deviance. In D. R. Laws \& W. O’Donohue (Eds.), Sexual deviance: Theory, assessment, and treatment (pp. 1-21). New York: Guilford Press.

16. Malamuth, N. M., 1989. The attraction to sexual aggression: Part One. Journal of Sex Research, 26, 26-49.

17. McGuire, R. J., Carlisle, J. M., \& Young, B. G., 1965. Sexual deviation as a conditioned behavior: A hypothesis. Behavior Research and Therapy, 2, 185-190.

18. Money, J., 1984, Paraphilias: Phenomenology and classification, American Journal of Psychotherapy, 38(2), 164-179.

19. Paulauskas, R., 2013. Is causal attribution of sexual deviance the source of thinking errors?. International Education Studies, Vol. 6(4).

20. Saleh, F.M. \& Berlin, F.S., 2008. Sexual deviancy: diagnostic and neurobiological considerations, Journal of Child Sexual Abuse, 12:3-4, 53-76. 
21. Sbraga, T. P., 2003. Sexual deviance and forensic psychology: a primer, Handbook of Rorensic Psychology, 429-470.

22. Scott, G. G., 1983. Dominant women, submissive men. New York: Praeger.

23. Simon, W. and J. Gagnon, 1967. 'Homosexuality: The Formulation of a Sociological Perspective',
Journal of Health and Social Behavior 8(3): 17785.

24. Spengler, A., 1977. Manifest sadomasochism of males: Results of an empirical study. Archives of Sexual Behavior, 6, 441-456.

25. Thornton, D., 1993. Sexual deviancy. Current Opinion in Psychiatry, 6, 786-789. 